\title{
How Athila retrotransposons survive in the Arabidopsis genome Antonio Marco ${ }^{1}$ and Ignacio Marín*2
}

\author{
Address: ${ }^{1}$ Center for Evolutionary Functional Genomics, The Biodesign Institute, Arizona State University, USA and ${ }^{2}$ Instituto de Biomedicina de \\ Valencia (CSIC), Valencia, Spain \\ Email: Antonio Marco - amarcoca@asu.edu; Ignacio Marín* - imarin@ibv.csic.es \\ * Corresponding author
}

Published: 14 May 2008

BMC Genomics 2008, 9:219 doi:10.1/86/147|-2164-9-219

This article is available from: http://www.biomedcentral.com/147I-2164/9/219

(c) 2008 Marco and Marín; licensee BioMed Central Ltd.

This is an Open Access article distributed under the terms of the Creative Commons Attribution License (http://creativecommons.org/licenses/by/2.0), which permits unrestricted use, distribution, and reproduction in any medium, provided the original work is properly cited.
Received: 5 December 2007

Accepted: 14 May 2008

\begin{abstract}
(1)
Abstract

Background: Transposable elements are selfish genetic sequences which only occasionally provide useful functions to their host species. In addition, models of mobile element evolution assume a second type of selfishness: elements of different familes do not cooperate, but they independently fight for their survival in the host genome.

Results: We show that recombination events among distantly related Athila retrotransposons have led to the generation of new Athila lineages. Their pattern of diversification suggests that Athila elements survive in Arabidopsis by a combination of selfish replication and of amplification of highly diverged copies with coding potential. Many Athila elements are non-autonomous but still conserve intact open reading frames which are under the effect of negative, purifying natural selection.
\end{abstract}

Conclusion: The evolution of these mobile elements is far more complex than hitherto assumed. Strict selfish replication does not explain all the patterns observed.

\section{Background}

Mobile elements are selfish genomic parasites that only rarely benefit their hosts [1-4]. They belong to two main classes, with or without RNA intermediates, and most eukaryotic genomes contain several types or families of elements of each class [5-7]. A family is a set of very similar sequences that generally include some active elements plus a variable number of non-autonomous, defective copies derived from the active ones. Classical mobile element evolution models suggested that selfishness drives the evolution of each family. Altruistically amplifying either defective copies or elements of other families would decrease the likelihood of long-term survival for a family of elements $[5,6,8]$. The available data for the Saccharomyces cerevisiae and Drosophila melanogaster genomes, among others, in which the rule is to find families of recent ori- gin, composed by almost identical and highly active elements $[9,10]$, agrees well with those models. However, whether elements that pervade other genomes, especially those with larger amounts of repetitive sequences, follow the same dynamics has been less extensively studied. In fact, the replication of some types of non-autonomous sequences (e. g. SINEs, MITEs, probably several types of retrotransposon-derived plant repeats) present in large numbers in some genomes depend on mobile elements (reviewed in [11-13]). It is not obvious what kind of advantage may obtain the mobile elements involved, and therefore those non-autonomous sequences are considered to replicate parasitically. However, it is possible to envisage situations in which the amplification of nonautonomous elements contributes to the survival of active elements, a possibility that remains largely unexplored. 
Some evidence for such type of cooperation within a family is available. For example, active Drosophila P elements may improve their likelihood of survival by replicating particular types of defective elements that negatively control the transposition rates of the active ones, thus diminishing the harmful effects on the host (reviewed in [14]; see also [15] for related examples).

Athila is one of the best-known plant long-terminalrepeat (LTR) retrotransposons [16-20]. It belongs to the Ty3/Gypsy group, evolutionary closely related to mammalian retroviruses [21]. Actually, some Athila retrotransposons and a few related plant elements are structurally identical to simple retroviruses. They have, in addition to their gag and pol genes, a third ORF, generally absent in other LTR retrotransposons. It may encode an envelope (Env) protein, potentially able to allow the generation of viral infective particles $([17,19,20]$; see review [22]). However, whether Athila behaves as an infective retrovirus is still unknown. The evolution of Athila retrotransposons has been traced back using phylogenetic analyses based on their reverse transcriptase (RT) sequences, which are part of the pol gene $[17,18,20,22,23]$. These analyses demonstrated that Athila elements are highly heterogenous. Particularly, our group showed that Athila RTs are more variable than those of other eight lineages of Arabidopsis Ty3/Gypsy retrotransposons and that there is no relationship between the degree of similarity among elements and the pattern of presence or absence of env sequences, suggesting that Athila evolution follows a complex pattern [18].

In this study, we show that the combined analyses of Athila gag, env and pol sequences provides a novel view of the evolutionary forces acting on these retrotransposons in the Arabidopsis genome. We determine that most Athila elements lack pol sequences and therefore are non-autonomous. Some of these elements have however retained intact ORFs that encode for Gag and Env proteins. These ORFs are under the effect of negative, purifying selection and therefore they must be functional. Moreover, diversification and survival of Athila elements in Arabidopsis has often involved recombination among distantly-related elements. In one particular case, recombination involving non-autonomous elements has contributed to generate an active element that moreover has acquired a typical retroviral structure. These results are not compatible with the simplistic view of selfish amplification of independent Athila families.

\section{Results \\ Arabidopsis Athila elements can be divided into ancient families, many of them exclusively composed by non- autonomous elements}

As already indicated above, the evolutionary analyses performed so far on Athila elements have been focused on comparing RT sequences. However, when we deeply examined the diversity of Athila elements, we detected that the analysis of pol-derived sequences may offer at most a partial view of the patterns of evolution of these elements. We found that many Athila elements are characterized by either of two alternative structures, typical of non-autonomous retrotransposons: 1) LTRs plus a single ORF encoding Gag proteins, or, 2) LTRs plus two ORFs, encoding Gag and Env proteins. We also found that all potentially autonomous Athilas, those with pol sequences (including RTs), also have gag sequences, although they may or may not have env sequences.

These results led us to the idea of reassessing Athila evolution from the point of view of their gag sequences. We reasoned that gag sequences, common to all types of both complete and non-autonomous elements, would provide the most precise picture of the evolutionary history of Athila retrotransposons. We thus built phylogenetic trees based on Athila gag sequences. We must note here that in a previous study, based on RT sequences, Athila and the closest relative of Athila, the env-lacking retrotransposon that we named Little Athila [18] were confounded [20]. However, the recent addition of many novel sequences allowed us to confirm that Athila and Little Athila elements are not only often structurally different (Athilas often contain env sequences, while Little Athilas always lack env), but also possess very different sequences and thus are better defined as two different elements. Particularly, we found that they appear as two separate lineages not only in Arabidopsis, but also in species of the Brassica genus. This result demonstrates that Athila and Little Athila split at least 15-20 millions of years ago (our results are summarized in [22]). This result was also found by Zhang and Wessler [23] in their general comparison of the elements present in Arabidopsis and Brassica. Those authors also considered Athila and Little Athila as two different elements. Thus, all the subsequent results shown here refer solely to Athila elements, as defined by Marín and Lloréns [18] and Zhang and Wessler [23].

Neighbor-joining and maximum parsimony phylogenetic trees based on gag sequences confirmed that Athila is a complex ensemble, formed by highly differentiated families. Twelve monophyletic, divergent Athila families became apparent in those trees (Figure 1; data for the elements can be found in [Additional file 1]). One of them ("Family 0" in Figure 1) included only highly defective copies and was not further analyzed. Table 1 contains the 


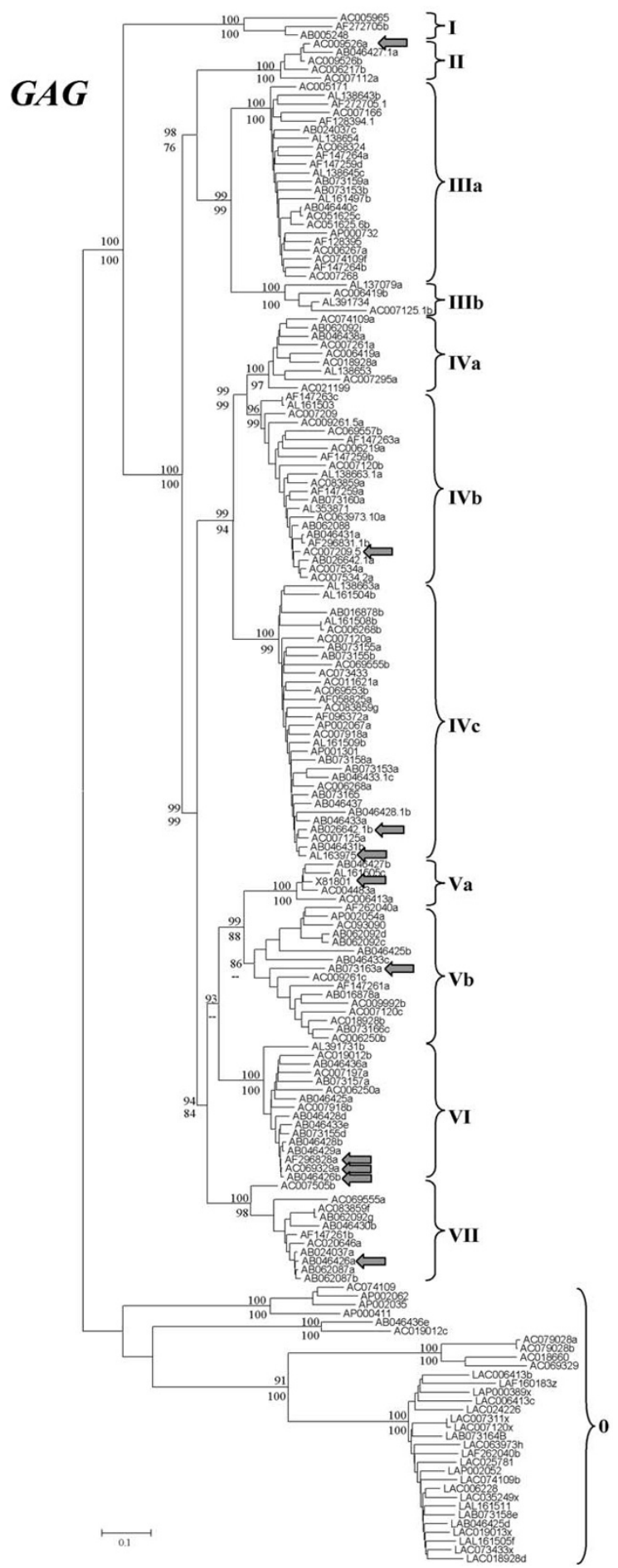

Figure I

Neighbor-joining phylogenetic tree based on the gag sequences of Arabidopsis thaliana Athila elements. The names refer to the accession numbers from which the elements were obtained. Some times, letters have been added at the end to name different elements present in the same sequence. Numbers in the branches refer to bootstrap support (in percentages) for two different methods, neighbor-joining (NJ, top) and maximum parsimony (MP, bottom). The results of both methods were, in this case and the ones in the next two figures, almost identical, so they can be shown in a single tree. Arrows points to the ten elements without frameshifts or stop codons in their ORFs (discussed in the text). 
Table I: Canonical Athila retroelements. The numbers refer to the nucleotides of each sequence that correspond to Athila ORFs or LTRs.

\begin{tabular}{|c|c|c|c|c|c|c|c|}
\hline \multirow[b]{2}{*}{ FAMILY } & \multirow[b]{2}{*}{ Acc. No. } & \multicolumn{4}{|c|}{ Locations of the ORFs } & \multirow[b]{2}{*}{ Locations of the LTRs } & \multirow[b]{2}{*}{ Insertion rangeł (Myr) } \\
\hline & & ORFI (gag) & $\begin{array}{c}\text { ORF2 (pol) } \\
\text { RT }\end{array}$ & $\begin{array}{l}\text { ORF2 (pol) } \\
\text { Integrase }\end{array}$ & ORF3 (env) & & \\
\hline $\mathbf{I}$ & AB005248.I & $26036-27931$ & $28901-29497$ & $30719-31504$ & $32343-33455$ & $24|25-257| 5 / 34256-35848$ & $0.07 \pm 0.03-1.60 \pm 0.33$ \\
\hline II & $A C 007 I 12.6$ & $13686-15547$ & $x$ & $\mathrm{X}$ & $17353-18318$ & ||87|-|3507/20699-22334 & $0.33 \pm 0.10-1.63 \pm 0.20$ \\
\hline IIIa & AC007268.5 & $54217-56048$ & $x$ & $x$ & $58170-59432$ & $52396-53654 / 61662-62920$ & $0.80 \pm 0.13-2.07 \pm 0.33$ \\
\hline IIIb & AC0064I9.4a & $16642-18676$ & $19664-20167$ & $2|48|-2226 \mid$ & $22764-24068$ & |4874-16490/25936-27580 & $1.77 \pm 0.20-2.07 \pm 0.23$ \\
\hline IVa & AC0064l9.4b & $77199-79245$ & $\mathrm{x}$ & $\mathrm{x}$ & $*$ & $75761-77058 / 82966-84163$ & $1.00 \pm 0.13-2.40 \pm 0.23$ \\
\hline IVb & AC007209.6 & $20563-|853|$ & $17510-16914$ & | $5695-14953$ & $13758-11905$ & 22465-20708/I0282-857। & $0.17 \pm 0.07-1.07 \pm 0.13$ \\
\hline IVc & $A C 007125.1$ & $8267-10612$ & $\mathrm{x}$ & $\mathrm{x}$ & $12350-14105$ & $6319-8119 / 15919-17719$ & $0.20 \pm 0.10-1.63 \pm 0.20$ \\
\hline $\mathbf{V a}$ & X81801.I & $1732-4539$ & $x$ & $x$ & $5248-7332$ & $1-1539 / 8954-10505$ & $0.07 \pm 0.03-1.73 \pm 0.23$ \\
\hline Vb & AF262040.I & $39443-41436$ & $x$ & $x$ & $*$ & $38797-39257 / 45690-46 \mid 43$ & $0.80 \pm 0.13-2.33 \pm 0.33$ \\
\hline VI & AC069329.6 & $4289 \mid-40537$ & $x$ & $x$ & $38906-36963$ & $44864-43063 / 35330-33522$ & $0.20 \pm 0.10-1.83 \pm 0.23$ \\
\hline VII & AB062087.I & $46573-44021$ & $43484-42995$ & $4|773-4099|$ & $*$ & $48228-46745 / 38467-37031$ & $0.17 \pm 0.07-0.93 \pm 0.13$ \\
\hline
\end{tabular}

*These elements contain a very short fragment of env, not long enough as to be included in the sequence alignments.

$\ddagger$ Range of insertion times detected within each family, estimated according to LTRs sequence similarity. In millions of years.

description of canonical copies for the other 11 families. Apart from the gag sequences of different families being very different, further demonstration for the high degree of diversification among Athila lineages was provided by the fact that LTRs of elements of different families were in general highly divergent and only partially alignable. We obtained estimates for the time when families diverged in the only two cases in which the full sequences of their LTRs could be compared (see Methods). Thus, families IVa and IVb were estimated to split about 2.7 millions of years ago, while families IIIa and IIIb diverged about 3.0 millions of years ago. These results agree well with the upper estimates for the time of insertion within a family, again according to LTR divergence, which reaches 2.4 millions of years (Table 1). If we now extrapolate from the data shown in Figure 1, assuming that divergence in gag sequences is roughly proportional to divergence time, an age of 6 to 10 millions of years for the most ancient splits among Athila families can be estimated. Thus, we can conclude that Athila families, as defined according to gag sequences, are ancient evolutionary lineages. An upper limit of less than 15-20 millions of years can be deduced from the fact that these eleven Arabidopsis families appear as a monophyletic group separated from all Brassica Athilas (data not shown).

Most significantly, only four of the eleven gag-defined families (I, IIIb, IVb and VII) contained elements with pol sequences (see Table 1). These results suggest that most Athila elements, in fact complete families, are non-autonomous, and that they propagate by using the enzymatic machinery provided by elements of other families. Comparative analyses of LTRs demonstrated that non-autonomous families have been multiplying in the genome for periods of time of up to 2 millions of years (Table 1 ). Obviously, these results also show that all accounts of
Athila element evolution published so far, based on RT sequences, offered a very incomplete view of the evolutionary dynamics of this complex ensemble of retroelements.

\section{Activity of Athila retrotransposons}

If we assume that the available sequences correctly represent the diversity of the Athila elements present in Arabidopsis thaliana, we may infer the degree of activity of the different families of elements by their number of active copies. Significantly, most Athila sequences are non functional. Out of the almost 200 sequences of Athila elements analyzed, we detected only 10 potentially active elements, which contained ORFs without any frameshifts or stop codons. These elements also contain all characteristic conserved amino acids of Athila Gag proteins and, those that contain pol sequences, also contain the typical motifs of the active centers of reverse transcriptases and integrases. The 10 elements belonged to seven different families, as follows: 1 element from family II, 1 element from family IVb, 2 elements from family IVc, 1 element from family $\mathrm{Va}, 1$ element from family $\mathrm{Vb}, 3$ elements from family VI and 1 element from family VII codons (see arrows in Figure 1). Interestingly, no element in four of those families (II, IVc, Va and VI) has pol sequences, they just contain gag or gag + env sequences. Thus, only three copies among all Athila elements found so far are potentially autonomous, pol-containing copies. Two of them are from family IVb - corresponding to the "Athila4" element already characterized as potentially autonomous by Marín and Lloréns [18] and Wright and Voytas [20] - and family VII, respectively. These two families contain other elements with pol sequences, albeit defective. The third one belongs, according to its gag sequence, to family $\mathrm{Vb}$, but, surprisingly, all but two elements in this family lack pol 
sequences. These peculiar elements, named Va-rec in Figure 1 , will be discussed in detail in the next section.

LTR comparative analyses showed that the youngest elements in three of the families without potentially active copies, IIIa, IIIb and IVa, retrotransposed 0.8, 1.8 and 1.0 millions of years ago respectively (Table 1 ). This result may imply that these families are currently extinct. However, the presence of active Athila elements of these families in other Arabidopsis genomes cannot be excluded. For the fourth family without active copies (family I), a very recent insertion (estimated to have occurred $0.07 \pm 0.03$ millions of years ago; Table 1) was detected, suggesting that this family is still active. On the other hand, the most recent copies of the families with potentially active elements are in general quite young (average: $0.28 \pm 0.09$ millions of years) suggesting that most or perhaps all of them are still currently replicating.

The low number of potentially active copies and of recently inserted retrotransposons suggest that Athila elements, at least in the strains from which the examined sequences were derived, have in general a very low level of activity. We specifically searched for Athila cDNAs in order to obtain further evidence for the level of activity of these elements. A total of 169 ESTs corresponding to
Athila cDNAs were found in the NCBI EST database and 121 of them could be unambiguously assigned to one of the defined Athila families (Table 2). Of them, just 48 ESTs could be assigned to pol-containing elements. However, none of them derived from the pol gene of those elements. Moreover, only 2 ESTs derived from one of the potentially active elements described above (the one in sequence AL163975; family IVc). If we consider that finding ESTs does not necessarily mean that these elements can transpose, that most of these ESTs derive from experimental conditions in which Athilas are known to be derepressed (see review by [24]) and that the NCBI database currently contains 1.3 millions of ESTs (an average of more than 40 per gene), it is clear that the level of Athila transcription and in general its ability to replicate must indeed be very limited.

\section{Recombination among elements of distantly related families}

As just detailed, one of the potentially active elements (Va-rec) contained a gag sequence that was included in family Vb in our phylogenetic tree (Acc. No. AB073163; Figure 1). However, closer inspection of this particular copy demonstrated that it was not a typical $\mathrm{Vb}$ element. We found that, while no element in family $\mathrm{Vb}$ has $e n v$ sequences (Table 1), the Va-rec element contained an env

Table 2: Results of selective regime analyses. " $x "$, " $y$ " and "z" refer to the three elements analyzed, with "z" being the one with coding potential, " $y$ " a very close relative and " $x$ " a more distant relative. In all cases except Va-rec, all elements in each analysis belong to the same family. For Va-rec, elements of the two families that give rise to the element were used. In this case, the gag sequences were not

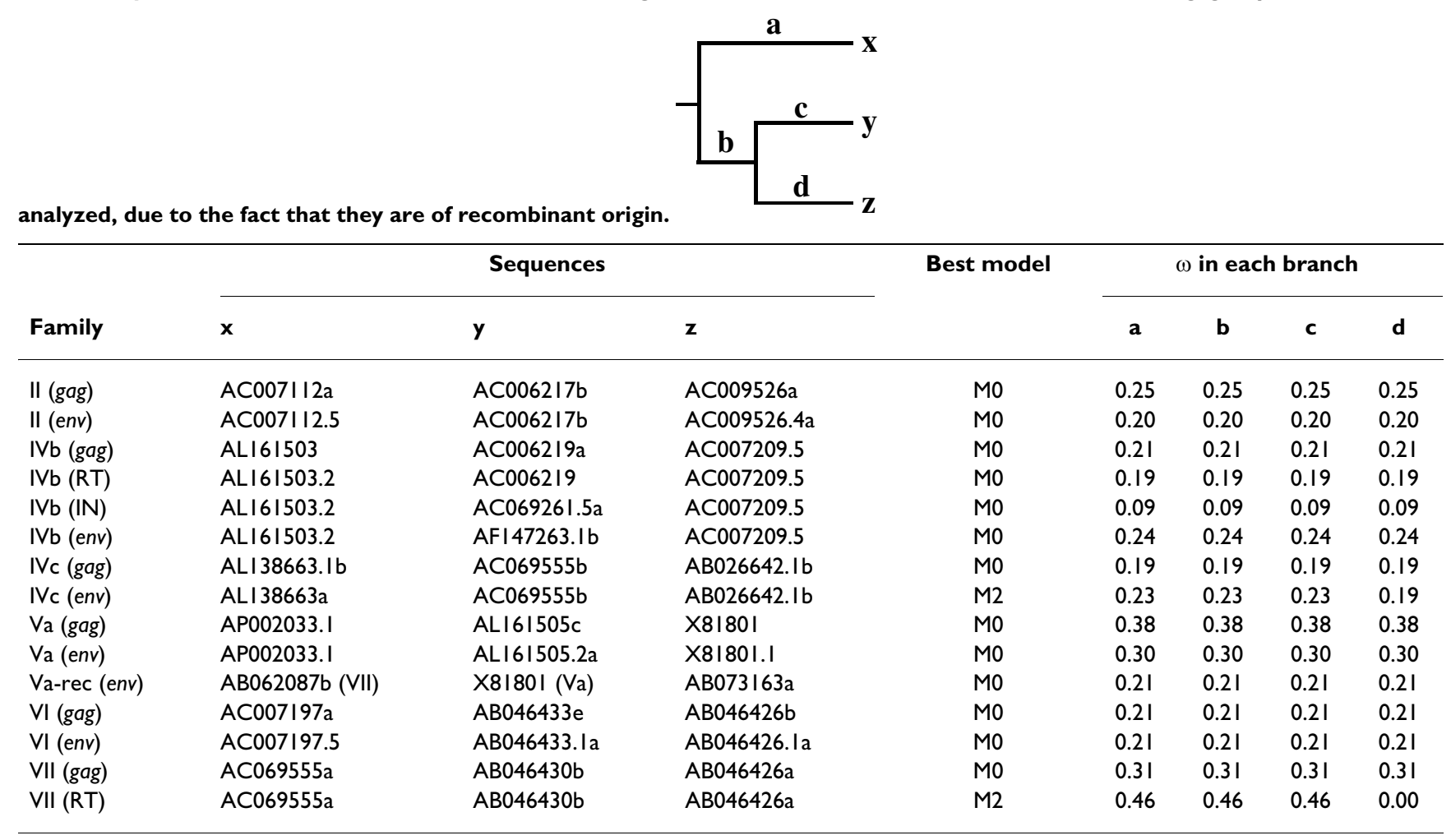


sequence very similar to those found in family Va. In addition and as already indicated above, Va-rec and another very similar but defective element (Acc. No. AB046433; Figure 1), were the only two elements having Vb-like gag sequences but containing also pol sequences. We noticed that these pol sequences were actually very similar to those found in family VII elements. These strange results, suggestive of a recombination process, led us to consider in more detail the relationships among gag, pol and env sequences for the whole set of Athila retrotransposons (see Figures 2, 3 and [Additional files 2, 3] for the details of the RT and $e n v$ sequences).

When trees generated with the gag (Figure 1), RT (polderived; Figure 2 and see [Additional file 2] for details) and env sequences (Figure 3 and [Additional file 3]) were compared, it was found that they were often congruent (Figures 4A, 4B). However, we observed several significant differences (outlined also in Figures 4A and 4B). They could only be explained by four independent recombination events among elements of distant families. Comparisons among LTRs and coding sequences allowed us to determine the evolutionary histories for those events:

1) Origin of Va-rec elements: We were able to determine that although these elements appeared in the gag-based phylogenetic trees as members of the $\mathrm{Vb}$ family, this was an artifact caused by them having a gag sequence of mixed origin. They emerged by the acquisition by an element of family Va of part of the gag gene and a complete pol gene derived from a family VII element (Figure 5A). Va-rec is therefore an element of recombinant origin, generated from a non-autonomous progenitor of the Va family that lacked pol. As we already mentioned, a second Va-rec element, but with stop codons and frameshifts was also detected. This finding demonstrates that Va-rec elements have been active after the recombination process that originated them. The only apparently active Va-rec element found has a relatively recent origin, becoming inserted $0.27 \pm 0.07$ millions of years ago.

2) Acquisition by a family IIIb element of $e n v$ sequences originated from a family Va element. This event explains the shift in the position of family IIIb elements in the gagand $e n v$-based trees (Figures 4A, 5B).

3) Recombination between elements of the IVc and VII families, to give rise to family VI. Family VI elements have LTRs and part of the gag sequences that are extremely similar to family VII elements, while the rest of the gag and the env sequences are very similar to those in family IVc (Figure 5C)

4) Acquisition of some family IIIa elements of an env of uncertain origin, generating an additional branch of ele- ments in the env-based tree, that we named IIIa-rec (Figure 5D).

In summary, these results demonstrate that recombination between elements of different families has occurred frequently in the past: at least 4 of the 13 lineages observed in this study (i. e. the 11 families described in Table 1 plus the IIIa-rec and Va-rec lineages, which cannot be detected in gag-based trees), are of recombinant origin. This is probably an understimate, because ancient recombination events or those involving short sequences would remain undetected with our methods. In any case, recombination has been so frequent that none of the phylogenetic trees obtained properly reflected the diversity of Athila retrotransposons. Only tree comparisons allowed us to understand the evolution of these elements.

\section{Selective pressures acting on Athila retroelements}

The fact that $70 \%$ of the potentially active copies encode for Gag or Env proteins but not for Pol proteins raises the question of whether the pol-less elements are simply parasites of the pol-containing copies or, alternatively, they may be contributing to their own propagation or to the propagation of other Athila elements. This contribution would require the production of active Gag or Env proteins by the non-autonomous elements. Of course, to conclude that these elements may contribute functional proteins is not enough to find out that the non-autonomous copies contain potentially coding ORFs or finding ESTs derived from these elements. Even then, all they could be propagating strictly in a parasitic way, i. e. depending solely on proteins provided in trans by other elements with their own genes being non-functional or fully repressed.

The question of whether particular elements contain functionally relevant sequences can be tackled by considering the selective pressures acting on them. Particularly, if they are functionally irrelevant, we would expect the coding regions of non-autonomous copies to evolve at a neutral rate. If, on the other hand, we find that evolution in certain coding regions of non-autonomous copies are under negative, purifying selection, then this would be a strong evidence for them being functionally significant. However, a problem that arises in this type of studies is the potential confusion caused by the past effects of negative selection. Imagine that, after an element replicates, there follows a period of time in which the coding sequences of both copies remain active. In that period, selection on their coding regions would generate an accumulation of changes in permissive positions (e. g. third positions of codons) respect to more constrained positions. If later one of these copies becomes inactive, and starts evolving neutrally, changes will accumulate at random. However, if, after some time, we compare both sequences, we may still 
$R T$

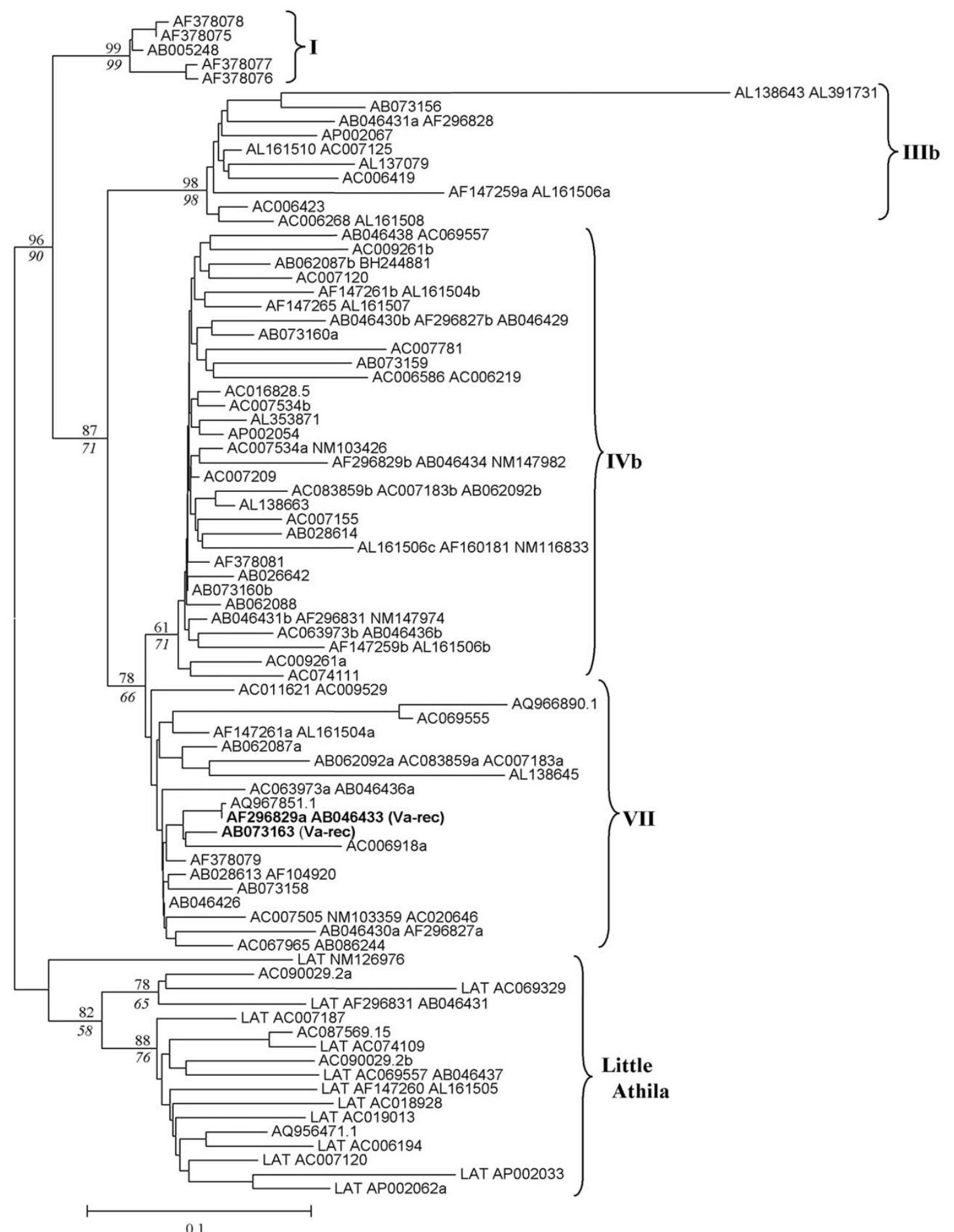

Figure 2

Neighbor-joining tree obtained for RT sequences of Athila elements in Arabidopsis thaliana. Names and bootstrap values (NJ/MP) as in Figure I. 


\section{ENV}

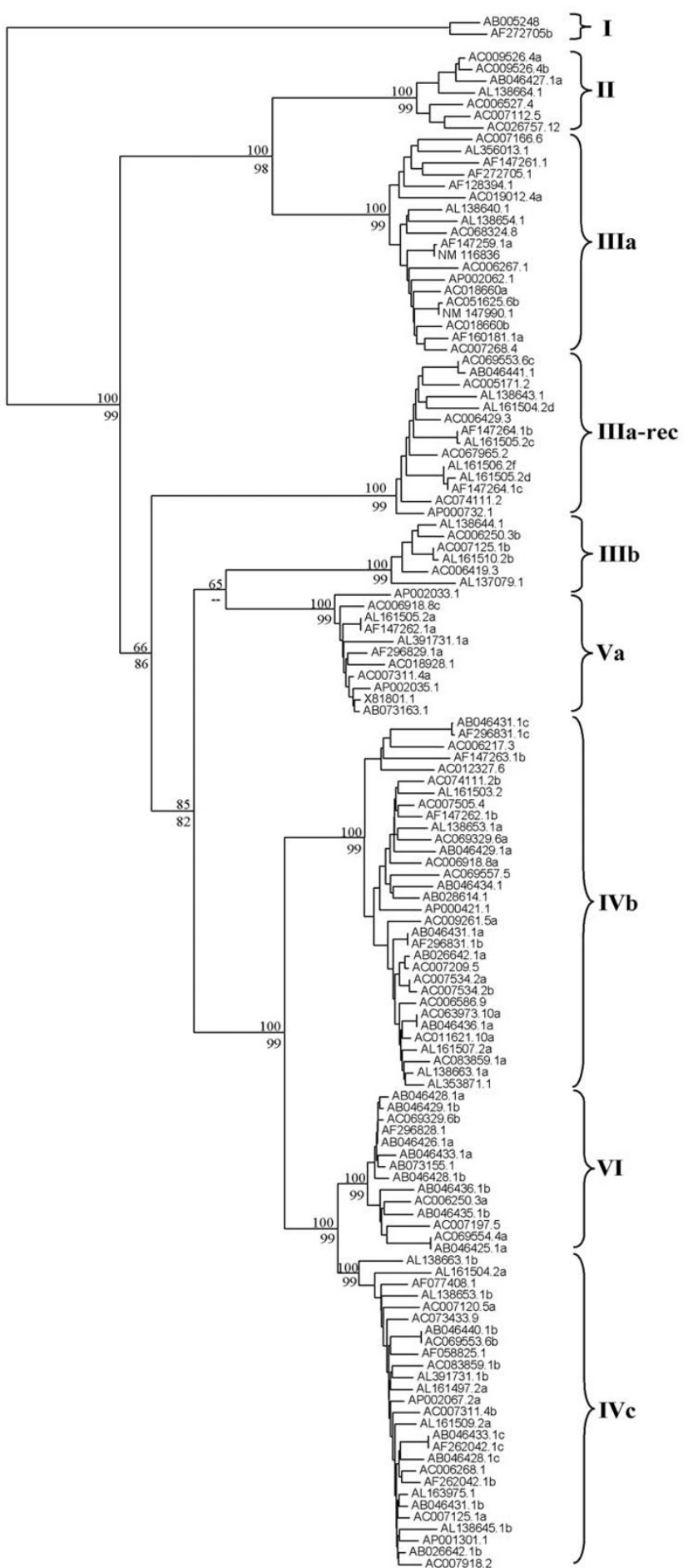

$\longmapsto$

Figure 3

Neighbor-joining tree based on env sequences of Arabidopsis thaliana Athilas. Names and bootstrap values again NJ/ $M P$ as in Figure I. 

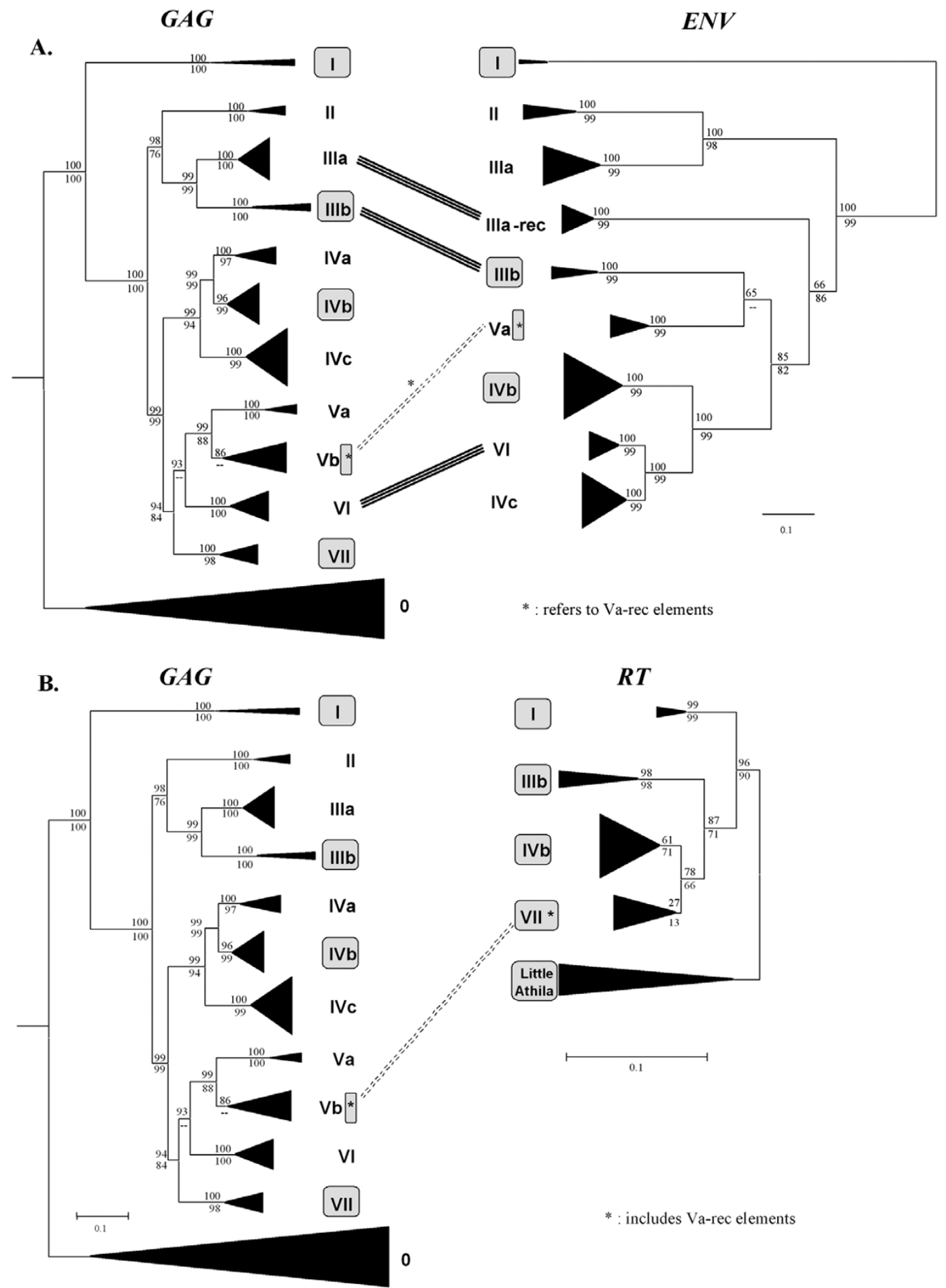

Figure 4

Comparisons of the phylogenetic trees obtained using different Athila coding sequences. A) Comparison of the Gag and Env trees. Lines connecting both trees indicate different relative positions of families or elements, corresponding to recombination events. The dashed lines refer to the recombination event that generated the Va-rec elements. Bootstrap support for the topology is shown in percentages (Top: neighbor-joining; Bottom: maximum parsimony). In gray boxes, we show the families that contain pol sequences. B) Comparison of the Gag and RT trees. 

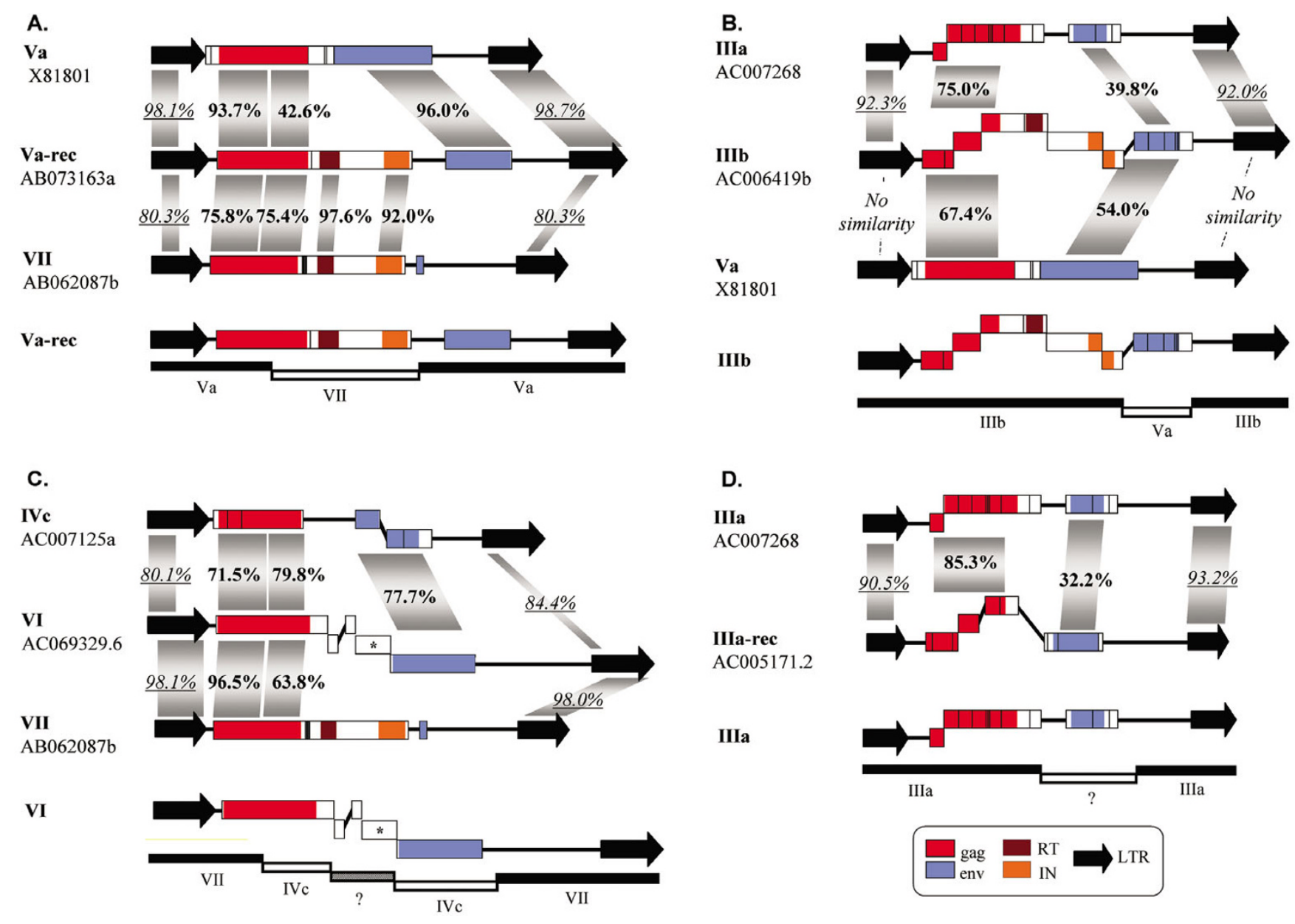

\section{Figure 5}

Recombination events. For panels $A$ to $D$, we show on the top the similarity between a canonical member of each recombinant lineage and the canonical members of the parental lineages that participated in the recombination event. On the bottom, we summarize the most likely origin of the different parts of the recombinant elements. Numbers in bold: percentage of amino acidic similarity. Numbers in italics and underlined: percentage of nucleotide identity. See details in the text.

find evidence for negative selection, due to the imprint of pass negative selection being still detectable. To avoid this problem, we followed the strategy of comparing the ORFs of potentially active Athila elements only with copies that were very closely related. More precisely, we analyzed trios of sequences, consisting on the sequence that we want to analyze, one of its closest relatives and a third, more distant relative. With these trios, for which phylogenetic relationships are obvious, we performed codon-based analyses of the relevant ORFs to establish the specific rates of non-synonymous vs. synonymous changes $(\omega)$ for the different branches (see details in Methods). All the relevant results are shown in Table 3 and the whole set of analyses is detailed in [Additional file 4]. The summary of these results is very simple: in all cases, the best model implies strong negative selection on the branch that corresponds to the elements with potentially active ORFs (branch " $z$ " in the schematic tree shown at the top of Table 3). These results strongly reinforce the idea that these ORFs indeed encode functional proteins, which may contribute to either the replication of the copies that carry them or of other elements in trans.

\section{Discussion}

We may now recapitulate the observations described in the previous chapter. First, we have shown that Athila is composed by at least 11 different families, defined as monophyletic groups of closely related elements (Figure 1). Most of these families emerged in the distant past. We dated the splits between families as having occurred at least 2.7 millions of years ago. Even considering all types of elements, autonomous or not, Athilas are not present in large numbers. Our results agree very well with a previous estimation of about 200 structurally intact copies of 
Table 3: Summary of ESTs derived from Athila elements

\begin{tabular}{|c|c|c|}
\hline Family & Most similar genomic DNA & ESTs \\
\hline I & AF272705b (env) & EG477448, EG477439, EG477443, EG477438, EG477426 \\
\hline I & AB005248 (env) & EG477445 \\
\hline IIla & AC007I66.6 (env) & EG52617I, EG526175, EG526169, EG526179, EG526177, EG526176, EG526150 \\
\hline Illb & AC007I $25.1 \mathrm{~b}$ (env) & EG4629II, EG455888, EG462908 \\
\hline Illb & AL391734 (env) & EG462905, EG462904, EG455886 \\
\hline IIlb & ALI37079.I (env) & 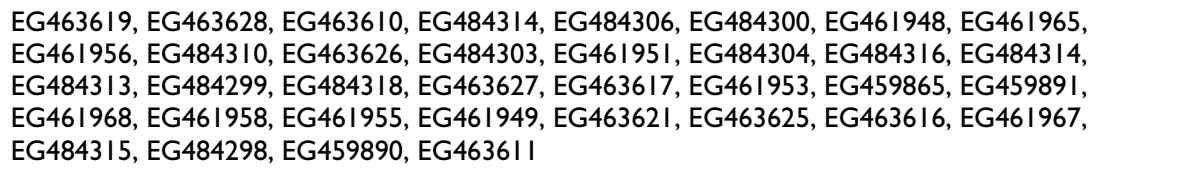 \\
\hline IVc & AB046427.la (gag) & $\begin{array}{l}\text { EG452894, EG452887, EG452883, EG452874, EG452872, EG452892, EG452885, EG452879, } \\
\text { EG452900, EG452890, EG452873, EG45287I }\end{array}$ \\
\hline IVc & AB046428.lb (gag) & BE526916 \\
\hline IVc & AP002067a (gag) & BP837984, BP837278 \\
\hline $\mathrm{Va}$ & ALI6I505.2a (env) & EG458656, EG458650 \\
\hline $\mathrm{Va}$ & AC004483a (gag) & $\begin{array}{l}\text { EG49|254, EG49|238, EG49|236, EG49|239, EG49|247, EG49|249, EG49|252, EG49|244, } \\
\text { EG49|246, EG49|253, EG49|242 }\end{array}$ \\
\hline $\mathrm{VI}$ & AB073157a (gag) & EG447|46, EG446|92, EG448096, EG4| 8344 \\
\hline VI & AB073I66.I (env) & EG526I58, EG526I52 \\
\hline $\mathrm{VI}$ & AB046428.Ib (env) & EG526117, EG526154, EG526119 \\
\hline VI & AB046425.I (env) & $\begin{array}{l}\text { EG479658, EG504857, EG4796I7, EG504777, EG479662, EG479648, EG479652, EG479650, } \\
\text { EG479663, EG4796II, EG479654, EG479607, EG504604, EG504604, EG479605, EG479657, } \\
\text { EG504582, EG479655, EG479604, EG479649, EG479646, EG479644, EG47966I, EG504856, } \\
\text { EG479643, EG47965I, EG479608 }\end{array}$ \\
\hline unassigned & - & $\begin{array}{l}\text { EG472931, EG47293।, EG459693, EG45969I, BP823996, BP822138, BP8I9I07, BP826056, EG49I235, } \\
\text { EG49124I, EG423786, EG49I243, EG49I248, EG49I250, EG49I245, EG49I237, EG459228, } \\
\text { EG459225, EG479658, EG504857, EG4796I7, EG504777, EG479662, EG479648, EG479652, } \\
\text { EG479650, EG479663, EG4796II, EG479654, EG479607, EG504604, EG479605, EG479657, } \\
\text { EG504582, EG479655, EG479604, EG479649, EG479646, EG479644, EG47966I, EG504856, } \\
\text { EG479643, EG47965I, EG479608, EG459688, EG459694, EG459692, EG459690 }\end{array}$ \\
\hline
\end{tabular}

Athila per genome [25]. There are no predominant families, so the number of elements for each family is low, ranging from 3 to 31 in our dataset and with an average of $13.2 \pm 2.8$ (see also Figure 1). Finally, there are only 3 potentially autonomous, active copies in the whole dataset. All these results, together with the low number of ESTs found, suggest that Athila activity is very low. If our results can be extrapolated to other Arabidopsis genomes, we can conclude that Athila as a whole is not a particularly succesful parasite, i. e. it survives at low numbers, and that individual Athila families are at the verge of extinction, at least in individual genomes (although perhaps they are doing fine in the whole species).

Our second main result is that we have shown that families that contain very similar elements without pol sequences have been replicating in the Arabidopsis genome for more than 2 millions of years. These elements are characterized by containing gag or gag + env sequences and several copies have kept apparently intact ORFs with coding potential and which are under a purifying selection regime. Thus, the proteins derived from elements of these families may be contributing to its own replication or to the replication of other Athila elements. Finally, the third main result is that recombination between elements of distantly related families is relatively frequent.

These results are quite different from those observed for most other LTR retrotransposons. For example, in the thoroughly analyzed Saccharomyces cerevisiae, Caenorhabditis elegans or Drosophila melanogaster genomes, most LTR retrotransposons are active, and there are no descriptions of abundant non-autonomous copies with coding potential $[9,10,26]$. Recombination between elements of different families of LTR retrotransposons (Ty1 and Ty2) leading to the generation of a new lineage (Ty1/Ty2) was first observed in S. cerevisiae $[27,28]$. However, differently for what we have found for recombinant Athilas, all Ty1/ Ty2 recombinant copies are recent [29] so their long-term evolutionary potential is unclear. Similar cases, in which new lineages of active elements are produced by recombination, have been described in other species [30,31]. Finally, a case in which a novel non-autonomous element that retains coding potential has emerged by recombination has been described in Hordeum [32,33], but, again, this element is very young and therefore their ability to propagate for long periods of time is unknown. Significantly, recombination leading to ORFs encoding for "hybrid" proteins of mixed origin, as occurred in Athila 
families Va-rec and VI (Figures 5A, 5C) was not found in any of these cases.

We may now ask what are the evolutionary processes that explains the particular pattern of evolution observed for Athila elements. First, we may consider whether our results are compatible with the hypothesis of full evolutionary independence of Athila families. To consider fully independent those families for which we have found only non-autonomous copies, we ought to hypothesize that hitherto undiscovered autonomous copies exist for those lineages. This is formally possible but very unlikely. These copies should be promoting the expansion of highly similar, structurally identical defective copies for periods of millions of years while not leaving any detectable pol-containing remnant in the genome. The best argument against this happening is that such peculiar pattern is never observed for the families that do have pol sequences. That is, although many copies in families with pol-containing elements are defective - having accumulated stop codons and frameshifts -, we never observed pol-less elements within those families. We may thus reason that, if in families for which pol-containing elements are known, we never detect a set of related pol-less elements, it is highly unlikely that precisely in those families for which we have not detected pol-containing elements, they actually exist. Therefore, the simplest explanation for the observed pattern is that pol-less elements are mobilized, at least in part, in trans, by enzymes provided by elements that belong to different, pol-containing, families.

We may then ask whether this is just another case of parasitism in which non-autonomous copies use the enzymatic machinery of the active ones without providing any compensation or, alternatively, some kind of cooperation between autonomous and non-autonomous elements might exist. There are two ways in which such cooperation may arise. First, non-autonomous elements with coding potential could contribute to the replication of autonomous copies. To demonstrate this process would require direct biochemical analyses, which is beyond the scope of this work. Our data show however that two necessary conditions for the process to occur are present: 1 ) there are non-autonomous elements with coding potential, with proteins which are under negative selective pressures; and, 2 ) the products of distant Athilas are biochemically compatible, as it is demonstrated by the emergence, by recombination, of new families with genes of different origin.

The second way in which cooperation might arise is indirect: generation of coding, non-autonomous copies could be advantageous for the long-term survival of Athila elements as a whole, if the non-autonomous copies occassionally contribute to the generation of novel successful families. Our results demonstrate that this type of event has occurred. We have shown that Athila autonomous and non-autonomous families are linked by recombination events and that several successful recombinant Athila lineages, defined as lineages able to replicate and survive for long periods of time, have arisen. They are of three different types: 1) novel autonomous lineages such as the Varec elements; 2) non-autonomous recombinant lineages that have survived while one or perhaps both progenitor families have become extinct, as seems to be the case for the family that provided the env sequences now found in IIIa-rec elements (Figure 5D); or, 3) simply recombinant non-autonomous lineages that are able to propagate in the genome as efficiently as autonomous ones (e. g. family VI, which has been replicating for at least two millions of years). Among all these results, it is most interesting that we may have detected the birth of a new evolutionary entity: if env sequences indeed provide Athila elements with the possibility of becoming infective, Va-rec elements would be an example of how recombination between an autonomous retrotransposon (env-less, from family VII) and a non-autonomous element (pol-less, from family Va) generates a novel active retrovirus (with gag, pol and env; Figure 5A). In any case, these events demonstrate that non-autonomous copies are not strictly parasitic. They are contributing to the long-term survival of Athila elements in Arabidopsis.

\section{Conclusion}

In summary, our results suggest that distant Athila families may be cooperating to survive in the Arabidopsis genome. Cooperation among other type of mobile elements, bacterial IS elements, has recently received attention, with the conclusion that it may appear under precise selective regimes [34]. Recent models also suggest situations in which mutualism may occur [35]. In fact, we think that the accepted view that all elements behave strictly selfishly may be due to the great difficulties involved in discovering patterns of sequence evolution compatible with cooperative processes. It is possible that other mobile elements follow dynamics similar to the one we have just described. For example, evidence for a related pattern of interchange to generate novel lineages is also available for human endogenous retroviruses ([36,37]; see also discussion in [38]). Therefore, this may be the first formal description of a widely-used survival strategy for eukaryotic mobile elements.

\section{Methods}

\section{Data mining and phylogenetic analyses}

We built databases of Gag, reverse transcriptase and Env proteins using BlastP and Tblast $\mathrm{N}$ searches against the databases available at the National Center for Biotechnology Information (NCBI). We used as queries multiple representative Athila elements until the searches become saturated. After each search, we aligned the sequences 
obtained and removed duplicates and partial sequences. All alignments were performed with ClustalX 1.83 [39] using default parameters. Alignments were manually corrected when necessary with GeneDoc 2.6 [40]. We used two methods of phylogenetic inference, neighbor-joining and maximum parsimony, implemented in MEGA2 [41] following the methods described in [42]. For both methods, statistical support for the branches was assessed performing 1000 bootstrap replicates.

\section{Structural analyses}

To determine the structure of Athila elements, we first used Blast2sequences searches [43], comparing each element with known gag, RT, integrase and env Athila sequences. ORFs were detected with ORF finder [44]. LTR locations were determined by looking for similarity within an element, also with Blast2sequences.

\section{Estimation of the insertion time or divergence time between elements}

We estimated the insertion time for an element or the divergence time for elements of two different families following the strategy described by San Miguel et al. [45]. The nucleotide sequences of either both LTRs of each element or single LTRs of two different elements were aligned and the Kimura two-parameter distance [46] was estimated using MEGA2. The distance obtained was then divided by two (because it refers to changes accumulated in both LTRs) and then again divided by the substitution rate at synonymous sites estimated for the brassicaceae Chs and Adh genes [47], that is, $1.510^{-8}$ per site per year.

\section{Characterization of recombination events}

Recombination events were deduced from incongruent phylogenetic positions of two proteins of a same element or group of elements [48]. We searched for the recombination breakpoints by analyzing pairwise alignments of amino acidic sequences and also, at the nucleotide level, following a sliding-window approach implemented in SimPlot [49], which utilizes the DNAPARS and NEIGHBOR programs of the Phylip package [50].

\section{Estimations fo synonymous and nonsynonymous nucleotide substitutions}

The rate of synonymous and nonsynonymous substitution were estimated using the PAML3.1 package [51], following the strategy of branch-dependent analyses described previously in [52]. Three models were analyzed. M0 refers to a model in which all branches are assumed to have the same rate. M1 is a model in which all branches are assumed to evolve at different rates. Finally, M2 is a model in which the branch that leads to the active element (" $z$ " in Table 2) is assumed to evolve at a different rate than the rest.

\section{Authors' contributions}

AM performed all the analyses presented here and contributed to the text. IM devised and supervised the research and wrote the manuscript.

\section{Additional material}

\author{
Additional file 1 \\ Supplementary_table_1 \\ Click here for file \\ [http://www.biomedcentral.com/content/supplementary/1471- \\ 2164-9-219-S1.pdf]
}

\section{Additional file 2}

Supplementary_table_2

Click here for file

[http://www.biomedcentral.com/content/supplementary/1471-

2164-9-219-S2.pdf]

\section{Additional file 3}

Supplementary_table_3

Click here for file

[http://www.biomedcentral.com/content/supplementary/1471-

2164-9-219-S3.pdf]

\section{Additional file 4}

Supplementary_table_4

Click here for file

[http://www.biomedcentral.com/content/supplementary/1471-

2164-9-219-S4.pdf]

\section{Acknowledgements}

Research supported by grant 200720102I (Proyectos intramurales especiales, CSIC. Spain).

\section{References}

I. Hurst GD, Werren JH: The role of selfish genetic elements in eukaryotic evolution. Nat Rev Genet 200 I, 2:597-606.

2. Kidwell MG, Lisch DR: Transposable elements as sources of genomic variation. In Mobile DNA II Edited by: Craig NL, Craigie R, Gellert M, Lambowitz AM. Washington: ASM Press; 2002:59-90.

3. Labrador M, Corcés V: Interactions between transposable elements and the host genome. In Mobile DNA II Edited by: Craig NL, Craigie R, Gellert M, Lambowitz AM. Washington: ASM Press; 2002:1008-1023.

4. Brookfield JFY: The ecology of the genome - Mobile DNA elements and their hosts. Nat Rev Genet 2005, 6:128-136.

5. Charlesworth B, Langley $\mathrm{CH}$ : The population genetics of Drosophila transposable elements. Annu Rev Genet 1989, 23:25I-287.

6. Charlesworth B, Sniegowski P, Stephan W: The evolutionary dynamics of repetitive DNA in eukaryotes. Nature 1994, 371:215-220.

7. Kazazian HHJ: Mobile elements: drivers of genome evolution. Science 2004, 303:1626-1632.

8. Kaplan N, Darden T, Langley $\mathrm{CH}$ : Evolution and extinction of transposable elements in mendelian populations. Genetics 1985, 109:459-480.

9. Lerat E, Rizzon C, Biémont C: Sequence divergence within transposable element families in the Drosophila melanogaster genome. Genome Res 2003, I3:1889-1896. 
10. Lesage P, Todeschini AL: Happy together: the life and times of Ty retrotransposons and their hosts. Cytogenet Genome Res 2005, I1 0:70-90.

II. Batzer MA, Deininger PL: Alu repeats and human genomic diversity. Nat Rev Genet 2002, 3:370-379.

12. Feschotte C, Jiang N, Wessler SR: Plant transposable elements: where genetics meets genomics. Nat Rev Genet 2002, 3:329-34 I.

13. Sabot F, Schulman AH: Parasitism and the retrotransposon life cycle in plants: a hitchhiker's guide to the genome. Heredity 2006, 96:38I-388.

14. Rio DR: $\mathbf{P}$ transposable elements in Drosophila melanogaster. In Mobile DNA I/ Edited by: Craig NL, Craigie R, Gellert M, Lambowitz AM. Washington: ASM Press; 2002:484-518.

15. Leonardo TE, Nuzhdin SV: Intracellular battlegrounds: conflict and cooperation between transposable elements. Genet Res 2002, 80:155-161.

16. Pélissier T, Tutois S, Deragon JM, Tourmente S, Genestier S, Picard $\mathrm{G}$ : Athila, a new retroelement from Arabidopsis thaliana. Plant Mol Biol 1995, 29:441-452.

17. Wright DA, Voytas DF: Potential retroviruses in plants: tat l is related to a group of Arabidopsis thaliana Ty3/gypsy retrotransposons that encode envelope-like proteins. Genetics | 49:703-7|5.

18. Marín I, Lloréns C: Ty3/gypsy retrotransposons: description of new Arabidopsis thaliana elements and evolutionary perspectives derived from comparative genomic data. Mol Biol Evol 2000, I7:1040-1049.

19. Vicient $\mathrm{CM}$, Kalendar R, Schulman $\mathrm{AH}$ : Envelope-class retroviruslike elements are widespread, transcribed and spliced, and insertionally polymorphic in plants. Genome Res 200I, I I:204|-2049.

20. Wright DA, Voytas DF: Athila4 of Arabidopsis and calypso of soybean define a lineage of endogenous plant retroviruses. Genome Res 2002, 12:|22-|3|.

21. Xiong $Y$, Eickbush TH: Origin and evolution of retroelements based upon their reverse transcriptase sequences. $E M B O$ 1990, 9:3353-3362.

22. Marco A, Marín I: Retrovirus-like elements in plants. Recent Res Devel Plant Sci 2005, 3:15-24.

23. Zhang X, Wessler SR: Genome-wide comparative analysis of the transposable elements in the related species Arabidopsis thaliana and Brassica oleracea. Proc Natl Acad Sci USA 2004 I OI:5589-5594.

24. Chan SW-L, Henderson IR, Jacobsen SE: Gardening the genome: DNA methylation in Arabidopsis thaliana. Nat Rev Genet 2005 , 6:35I-360.

25. Pereira $\mathrm{V}$ : Insertion bias and purifying selection of retrotransposons in the Arabidopsis thaliana genome. Genome Biol 2004, 5:R79.

26. Bowen NJ, McDonald JF: Genomic analysis of Caenorhabditis elegans revelas ancient families of retroviral-like elements. Genome Res 1999, 9:924-935.

27. Jordan IK, McDonald JF: Evidence for the role of recombination in the regulatory evolution of Saccharomyces cerevisiae Ty elements. J Mol Evol 1998, 47:14-20.

28. Jordan IK, McDonald JF: Phylogenetic perspective reveals abundant TyI/Ty2 hybrid elements in the Saccharomyces cerevisiae genome. Mol Biol Evol 1999, 16:419-422.

29. Jordan IK, McDonald JF: Tempo and mode of Ty element evolution in Saccharomyces cerevisiae. Genetics 1999, I5 I:I34I-I35I.

30. Kelly FD, Levin HL: The evolution of transposons in Schizosaccharomyces pombe. Cytogenet Genome Res 2005, I I 0:566-574.

31. Mugnier N, Biémont C, Vieira C: New regulatory regions of Drosophila 4 I 2 retrotransposable element generated by recombination. Mol Biol Evol 2004, 22:747-757.

32. Vicient $C M$, Kalendar $\mathrm{R}$, Schulman $A H$ : Variability, recombination, and mosaic evolution of the barley BARE-I retrotransposon. J Mol Evol 2005, 61:275-291.

33. Tanskanen JA, Sabot F, Vicient C, Schulman AH: Life without GAG: the BARE-2 retrotransposon as a parasite's parasite. Gene 2007, 390: 166-174.

34. Wagner A: Cooperation is fleeting in the world of transposable elements. PloS Comput Biol 2006, 2:e162.

35. Le Rouzic A, Dupas S, Capy P: Genome ecosystem and transposable element species. Gene 2007, 390:214-220.
36. Bénit L, Dessen P, Heidmann T: Identification, phylogeny, and evolution of retroviral elements based on their envelope genes. J Virol 2001, 75: II709-1 I7I9.

37. Jordan IK, McDonald JF: A biologically active family of human endogenous retroviruses (HERVs) evolved from an ancient inactive lineage. Genome Lett 2002, 2: 105-109.

38. Bannert $N$, Kurth $R$ : The evolutionary dynamics of human endogenous retroviral families. Annu Rev Genomics Hum Genet 2006, 7:149-173.

39. Thompson JD, Gibson TJ, Plewniak F, Jeanmougin F, Higgins DG: The CLUSTAL_X windows interface: flexible strategies for multiple sequence alignment aided by quality analysis tools. Nucleic Acids Res 1997, 25:4876-4882.

40. Nicholas KB, Nicholas HB: Genedoc: analysis and visualization of genetic variation. Distributed by the authors. 1997 [http:// www.genedoc.us]

4I. Kumar S, Tamura K, Jakobsen IB, Nei M: Mega2: molecular evolutionary genetics analysis software. Bioinformatics 200I, 17:1244-1245.

42. Marco A, Cuesta A, Pedrola L, Palau F, Marín I: Evolutionary and structural analyses of GDAPI, involved in Charcot-MarieTooth disease, characterize a novel class of glutathione transferase-related genes. Mol Biol Evol 2004, 2 I: I76- 187.

43. Tatusova TA, Madden TL: Blast 2 sequences, a new tool for comparing protein and nucleotide sequences. FEMS Microbio Lett 1999, 174:247-250.

44. ORF finder web page [http://www.ncbi.nlm.nih.gov/projects/gorf]

45. SanMiguel P, Gaut BS, Tikhonov A, Nakajima Y, Bennetzen JL: The paleontology of intergene retrotransposons of maize. Nat Genet 1998, 20:43-45

46. Kimura M: A simple method for estimating evolutionary rates of base substitutions through comparative studies of nucleotide sequences. J Mol Evol 1980, 16:1 II-120

47. Koch MA, Haubold B, Mitchell-Olds T: Comparative evolutionary analysis of chalcone synthase and alcohol dehydrogenase loci in Arabidopsis, Arabis, and related genera (brassicaceae). Mol Biol Evol 2000, 17:| 483-1498.

48. Posada D, Crandall KA, Holmes EC: Recombination in evolutionary genomics. Annu Rev Genet 2002, 36:75-97.

49. Lole KS, Bollinger RC, Paranjape RS, Gadkari D, Kulkarni SS, Novak NG, Ingersoll R, Sheppard HW, Ray SC: Full-length human immunodeficiency virus type I genomes from subtype c-infected seroconverters in india, with evidence of intersubtype recombination. J Virol 1999, 73:152-160.

50. Felsenstein J: Phylip (phylogeny inference package) version 3.5c. distributed by the author. Department of Genetics, University of Washington, Seattle; 1993.

51. Yang Z: Paml: a program package for phylogenetic analysis by maximum likelihood. Comput Appl Biosci 1997, 13:555-556.

52. Fares MA, Bezemer D, Moya A, Marín I: Selection on coding regions determined Hox7 genes evolution. Mol Biol Evol 2003, 20:2104-2II2.

Publish with Bio Med Central and every scientist can read your work free of charge

"BioMed Central will be the most significant development for disseminating the results of biomedical research in our lifetime. "

Sir Paul Nurse, Cancer Research UK

Your research papers will be:

- available free of charge to the entire biomedical community

- peer reviewed and published immediately upon acceptance

- cited in PubMed and archived on PubMed Central

- yours - you keep the copyright 